\title{
A TEST THEOREM ON COHERENT GCD DOMAINS
}

\author{
ZHAO YICAI \\ (Communicated by Maurice Auslander)
}

\begin{abstract}
Let $R$ be a commutative indecomposable coherent ring. Then the following statements are equivalent: (i) $R$ is a GCD domain; (ii) $R_{M}$ is a GCD domain for every maximal ideal of $M$ of $R$, and every finitely generated projective ideal in $R$ is principal; (iii) every two-generated ideal in $R$ has finite projective dimension, and every finitely generated projective ideal in $R$ is principal. Auslander-Buchsbaum's Theorem, etc. can be obtained from the result above.
\end{abstract}

Let $R$ be a commutative ring with $1 \neq 0$ in this paper. $R$ is called a GCD domain if every two elements $a, b \in R$ have a greatest common divisor (denoted by $[a, b]$ ) in $R, R$ is said to be indecomposable if 1 is the only nonzero idempotent of $R$. A coherent ring is a ring whose finitely generated ideals are finitely presented.

Definition. We say that $R$ has PPC if every finitely generated projective ideal in $R$ is a principal ideal.

Theorem. Let $R$ be an indecomposable coherent ring. Then the following statements are equivalent:

(i) $R$ is a GCD domain.

(ii) $R_{M}$ is a GCD domain for every maximal ideal $M$ of $R$, and $R$ has $P P C$.

(iii) Every two-generated ideal in $R$ has finite projective dimension, and $R$ has PPC.

We first quote two results from Vasconcelos [1] that will be fundamental to this note.

Lemma 1 [1, Corollary 5.16]. Let $R$ be a coherent local ring such that every principal ideal has finite projective dimension. Then $R$ is a domain.

Lemma 2 [1, Corollary 5.20]. Let $R$ be a coherent domain, and let $I$ be a finitely generated ideal of finite projective dimension. Then $I=d(R / I) J$, where $d(R / I)$ is an invertible ideal and $J$ is an ideal of $R$ satisfying $J^{-1}=R$.

Proof of Theorem. (i) $\Rightarrow$ (ii) Let $M$ be a maximal ideal of $R$. Let $\frac{a}{1}, \frac{b}{1} \in R$, and write $c=[a, b]$. if $\frac{d}{1}$ is a common divisor of $\frac{a}{1}$ and $\frac{b}{1}$ in $R_{M}$ then $d \mid s a$

Received by the editors November 6, 1990 .

1991 Mathematics Subject Classification. Primary 13C05, 13G05.

(c) 1992 American Mathematical Society $0002-9939 / 92 \$ 1.00+\$ .25$ per page 
and $d \mid t b$ for some $s, t \in R-M$. Thus $d$ is a common divisor of sta and $s t b$ in $R$. Since $s t c=[s t a, s t b]$, we have $d \mid s t c$. But $s t \notin M$. Hence $\frac{d}{1} \mid \frac{c}{1}$, that is, $\frac{c}{1}=\left[\frac{a}{1}, \frac{b}{1}\right]$. Therefore, $R_{M}$ is a GCD domain for every maximal ideal $M$ of $R$. Finally, let $I=\left(a_{1}, \ldots, a_{n}\right)$ be a finitely generated projective ideal of $R$. Then $I$ is an invertible ideal, that is, $I I^{-1}=R$. It is straightforward to check that $I^{-1}=\frac{1}{c} R$. Hence $I=c R$. Therefore $R$ has PPC.

(ii) $\Rightarrow$ (iii) Let $a, b \in R$. Consider the exact sequence

$$
0 \rightarrow(a) \cap(b) \rightarrow R^{2} \rightarrow(a, b) \rightarrow 0 .
$$

Let $M$ be any maximal ideal of $R$. Since $R_{M}$ is a GCD domain, $(a)_{M} \cap(b)_{M}$ is a principal ideal of $R_{M}$, and hence $(a)_{M} \cap(b)_{M}$ is 0 or $R_{M}$-free. It follows that $(a) \cap(b)$ is a flat ideal of $R$. But $(a) \cap(b)$ is finitely presented because $R$ is a coherent ring. Hence $(a) \cap(b)$ is projective, which shows that $p d_{R}(a, b) \leq 1$. Therefore, $R$ has PPC.

(iii) $\Rightarrow$ (i) We first prove that $R$ is a domain. Let $0 \neq a \in R$. Consider the exact sequence

$$
0 \rightarrow \operatorname{ann}(a) \rightarrow R \rightarrow a R \rightarrow 0 .
$$

Let $M$ be any maximal ideal of $R$. By Lemma 1, $R_{M}$ is a domain and hence $a R_{M}$ is 0 or $R_{M}$-free. Now $a R$ is a projective ideal of $R$, that is, $R=\operatorname{ann}(a) \oplus I$ for some ideal $I$ of $R$. But $R$ is indecomposable and $a \neq 0$. Hence $\operatorname{ann}(a)=0$ and $R$ is a domain.

Finally, let $a, b \in R$. We may assume that $a, b$ are nonzero and nonunit. Write $I=(a, b)$. By Lemma 2, $(a, b)=d(R / I)(u, v)$, where $(u, v)^{-1}=R$. Since $d(R / I)$ is an invertible ideal, $d(R / I)$ is finitely generated projective, and hence $d(R / I)=c R$ for some $c \in R$. Thus $(a, b)=c(u, v)$ and $a=c u$, $b=c v$. It is clear that $c=[a, b]$. Therefore, $R$ is a GCD domain.

A ring $R$ is called regular if every finitely generated ideal in $R$ has finite projective dimension.

Corollary 1 (Auslander-Buchsbaum's Theorem [2]). A Noetherian regular local ring is a unique factorization domain.

Corollary 2 (Vasconcelos's Theorem [1]). A coherent regular local ring is a GCD domain.

Corollary 3 (Kaplansky's Theorem [3]). Let $R$ be a Noetherian regular domain in which every invertible ideal is principal. Then $R$ is a unqiue factorization domain.

Proof. Noting that a local ring has PPC and that every projective ideal in a domain is invertible, we have Corollaries $1-3$ by the theorem.

Corollary 4. Let $R$ be a coherent local ring (or an indecomposable semilocal ring). Then $R$ is a GCD domain if and only if every two-generated ideal in $R$ has finite projective dimension.

Corollary 5. Let $R$ be an indecomposable coherent regular ring. Then $R$ is $a$ $G C D$ if and only if $R$ has PPC.

Corollary 6. Let $R$ be an indecomposable coherent ring in which every twogenerated ideal has finite projective dimension. Then $R$ is an integrally closed domain. 
Proof. First, $R$ is a domain (see the proof of (ii) $\Rightarrow$ (iii)). Second, $R_{M}$ is a GCD domain for every maximal ideal $M$ of $R$, and hence integrally closed. Therefore $R$ is integrally closed.

\section{REFERENCES}

1. W. V. Vasconcelos, The rings of dimension two, Dekker, New York, 1976.

2. M. Auslander and D. Buchsbaum, Unique factorization in regular local rings, Proc. Nat. Acad. Sci. U.S.A. 45 (1959), 733-734.

3. I. Kaplansky, Commutative rings, Allyn and Bacon, Chicago 1976.

Department of Mathematics, Guangxi Teachers University, Guilin 541004, China 